Communications in Physics, Vol. 22, No. 4 (2012), pp. 365-374

\title{
THE VATLY RADIO TELESCOPE
}

\author{
NGUYEN VAN HIEP, PHAM TUAN ANH, PHAM NGOC DIEP, \\ PHAM NGOC DONG, DO THI HOAI, PHAM THI TUYET NHUNG, \\ NGUYEN THI THAO, AND PIERRE DARRIULAT \\ VATLY, Institute for Nuclear Science and Technology
}

\begin{abstract}
A small radio telescope (SRT) has been installed on the roof of the Hanoi astrophysics laboratory VATLY. It is equipped with a $2.6 \mathrm{~m}$ diameter mobile parabolic dish remotely controlled in elevation and azimuth and with super-heterodyne detection around the $21 \mathrm{~cm}$ hydrogen line. First results of observations of the Sun and of the centre of the Milky Way are presented. They demonstrate the high quality of the telescope performance and are used to evaluate lobe size, signal to noise ratios, anthropogenic interferences and measurement accuracies. Particular attention is given to the measurement of the pointing accuracy. The rich measurement programme that is now at hand is briefly sketched.
\end{abstract}

\section{THE VATLY RADIO TELESCOPE}

A small radio telescope (SRT) has been installed on the roof of the VATLY astrophysics laboratory [1], commissioned and run-in. It is now routinely taking data. The present note presents its main features, illustrates its performance and provides evidence for excellent measuring conditions on both the continuum and the $21 \mathrm{~cm}$ hydrogen line.

The telescope is equipped with a mobile parabolic dish, $2.6 \mathrm{~m}$ in diameter, remotely adjustable in elevation and azimuth (Fig. 1). The reflected power is collected at the focus, where it is locally preamplified, shifted to lower frequency using standard superheterodyne, amplified and digitized. Standard data collection consists in a sequence of successive measurements of $\sim 7.7 \mathrm{~s}$ duration each, digitized in the form of a frequency histogram covering $\sim 1.2 \mathrm{MHz}$ in 156 bins of $\sim 7.8 \mathrm{kHz}$ each. Such a typical distribution is shown in Figure 2 (left). The $21 \mathrm{~cm}$ hydrogen line is clearly seen above a slowly varying continuum. The hydrogen line signals the presence of hydrogen clouds in the field of view and is associated with electron spin flip in the hydrogen atom. Galaxies such as ours contain many such clouds and the signal from the Milky Way is particularly strong. On the contrary, the continuum signals the presence of ionized matter and is associated with thermal or free-free (bremsstrahlung) emission.

The telescope orientation is remotely adjustable and a small TV camera allows watching the antenna movement from the control room below where a laptop displays the data being recorded and other relevant information. 


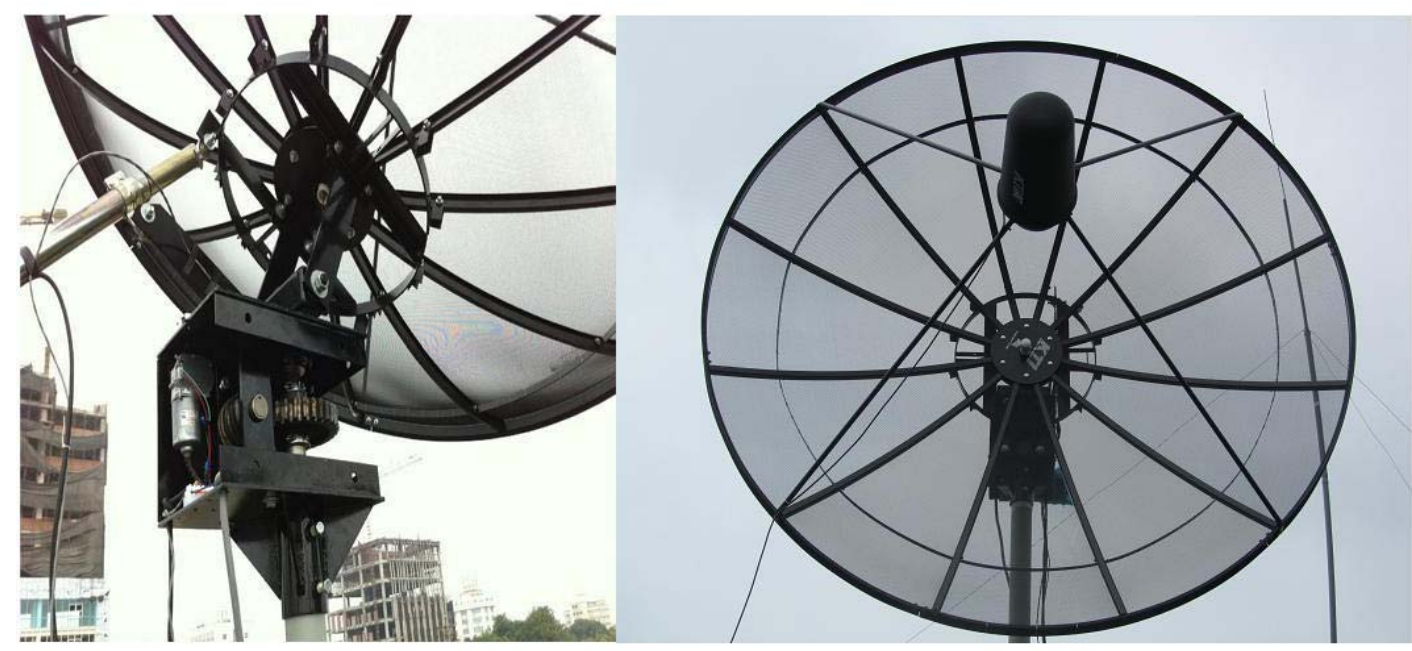

Fig. 1. Close-up views of the SRT antenna and of the motor system (gear box and telescopic arm, left panel) and of the feed horn and the calibration antenna (right panel).
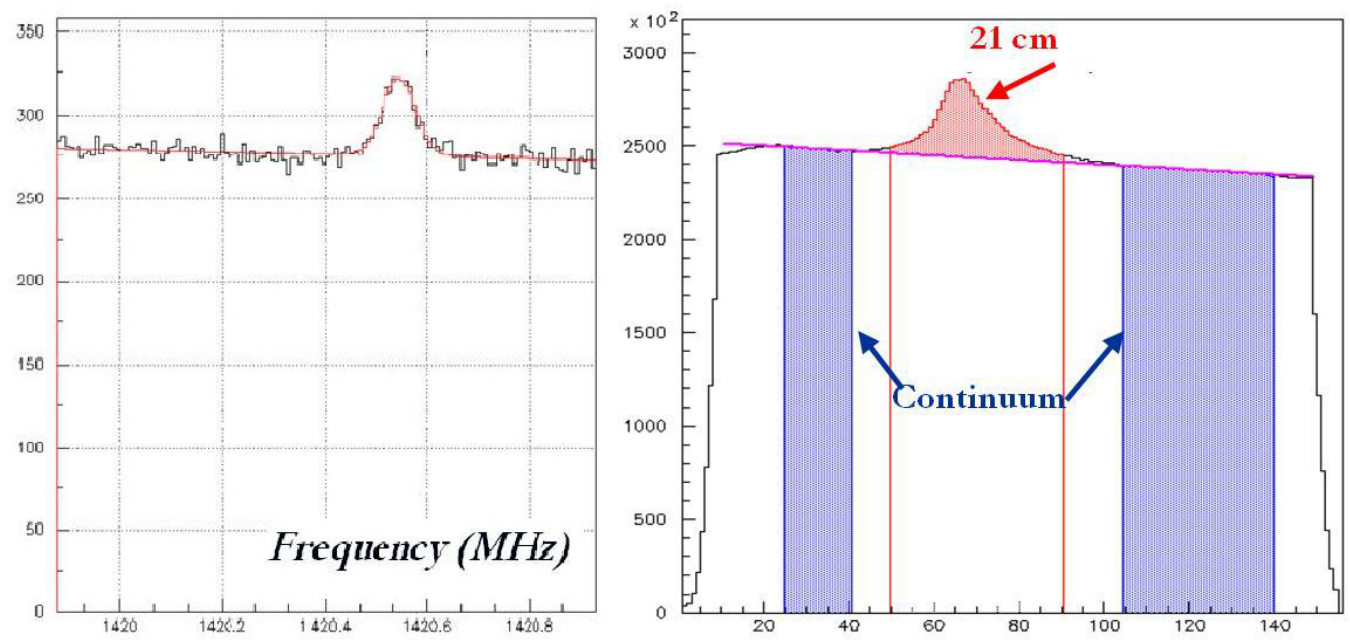

Fig. 2. A typical frequency spectrum (left) and its decomposition in $21 \mathrm{~cm}$ and continuum signals (right).

The following sections describe data collected on various targets including the Sun, with a strong continuum signal, and $\operatorname{SgrA}^{*}$ at the centre of the Milky Way, with a strong $21 \mathrm{~cm}$ signal. They are used to illustrate and quantitatively evaluate the performance of the telescope and to sketch a programme of future observations. A preliminary account of this work was presented at a recent conference [2]. 


\section{THE BACKGROUND SKY AND MEASUREMENT ACCURACY}

When pointing the telescope to a fixed direction in the sky, one records the sum of a general background and of signals associated with radio sources passing by as the Earth rotates (one speaks of drift scans). Examples of drift scans on the Sun and on Sgr A* respectively are illustrated in the next sections. In the present section, we are not interested in a particular radio source but rather in the contribution of the background. Collecting such data over several days shows the presence of spikes, sometimes occurring on a single day, sometimes repeating each day (Fig. 3, left). They are the result of interferences with anthropogenic parasites having their source in the severe electromagnetic pollution that exists above Hanoi and become more important at low elevations as can be expected.
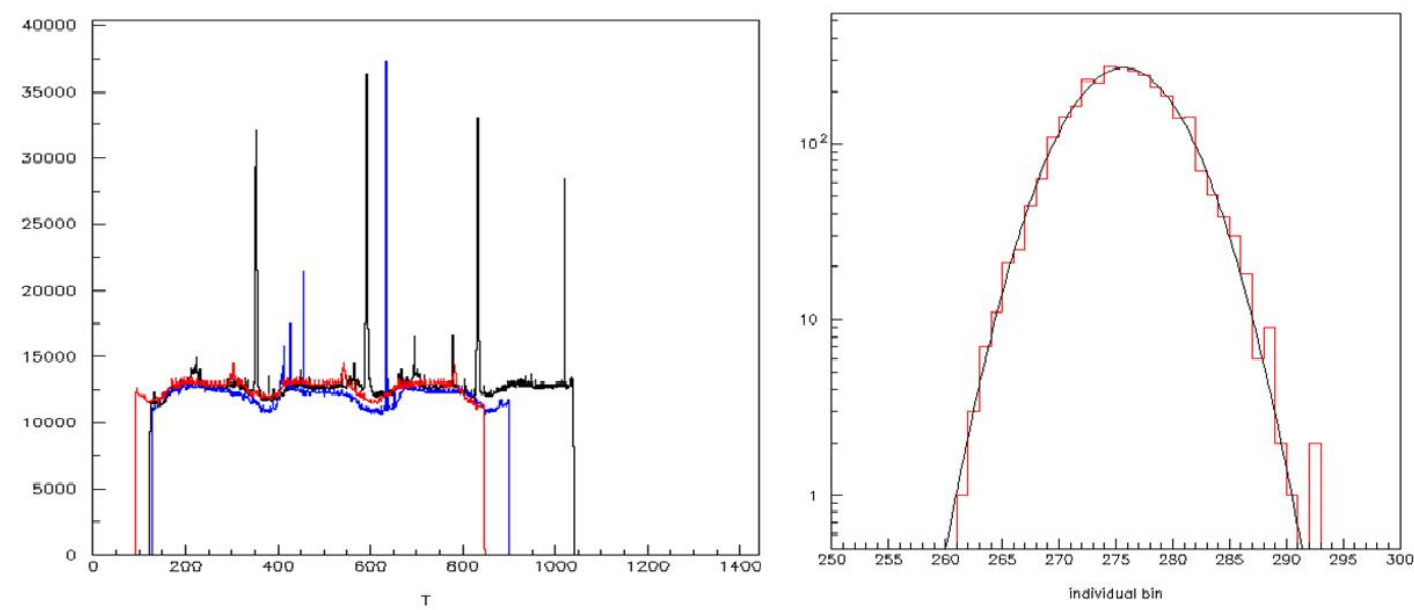

Fig. 3. Left: Time dependence of the content of bin 35. The abscissa is measured in tenths of an hour. The full range is nearly 5 days. Different curves correspond to different elevations. The first three large black spikes are due to the Sun passing by. Right: Distribution of measurements made in a single frequency bin during a stable period of $\sim 6.2$ hours after correction for slow drifts.

However, while such pollution prevents reliable detection of the Sun in the $50 \mathrm{~cm}$ range [3], it can be easily handled in the present frequency band (which is officially protected). In the following, such spikes are removed from the data and the frequency distributions that are shown are free of parasite interferences. In order to illustrate the stability of the response, we show in Fig. 3 (right) the distribution of the signal measured in a same frequency bin over a $\sim 6.2 \mathrm{~h}$ period (after correction for slow drifts). The fact that the distribution is well described by a Gaussian indicates that the measurement uncertainties are dominated by statistics. A systematic study of such data allows for an estimate of the relative measurement uncertainty obtained for a single frequency bin by summing $n$ successive measurements: $\Delta P / P=\sqrt{(0.27 \%)^{2}+(1.59 \%)^{2} / n}$. For $n \sim 35$, corresponding to $4.7 \mathrm{mn}$, the two terms under the square root are equal, giving an optimal time scale of, say, $\sim 10 \mathrm{mn}$ per measurement. The constant term, $\sim 0.3 \%$, is of systematic origin and 
essentially common to all frequency bins: it gives a measure of the best possible accuracy obtainable in measuring the power flux of a given radio source.

Absolute calibration of the power flux measurement can be done using a small reference dipole provided by the manufacturer but has not yet been studied with sufficient care to assess its reliability and accuracy.

\section{THE SUN: POINTING ACCURACY}

The Sun gives a strong signal in the continuum while the $21 \mathrm{~cm}$ line is essentially unaffected. As its apparent diameter is much smaller than the antenna lobe (the Sun seen at $21 \mathrm{~cm}$ is dominated by solar spots above a disk having the same size as the photosphere) the Sun can be considered as being a point source. In the present section we use it to assess the SRT pointing accuracy.

\section{III.1. Grid scans}

In order to reveal possible pointing errors, 26 grid scans (Fig. 4) have been made at different times of the day between October and December 2011. Each grid scan takes only $6 \mathrm{mn}$ and consists in 25 measurements pointing to the nodes of a $5 \times 5$ grid centred on the Sun and having a mesh size of $\sim 2.6^{\circ}\left(1 / 2\right.$ beam width) in elevation $h$ and $\sim 2.6^{\circ} / \cosh$ in azimuth $a$.

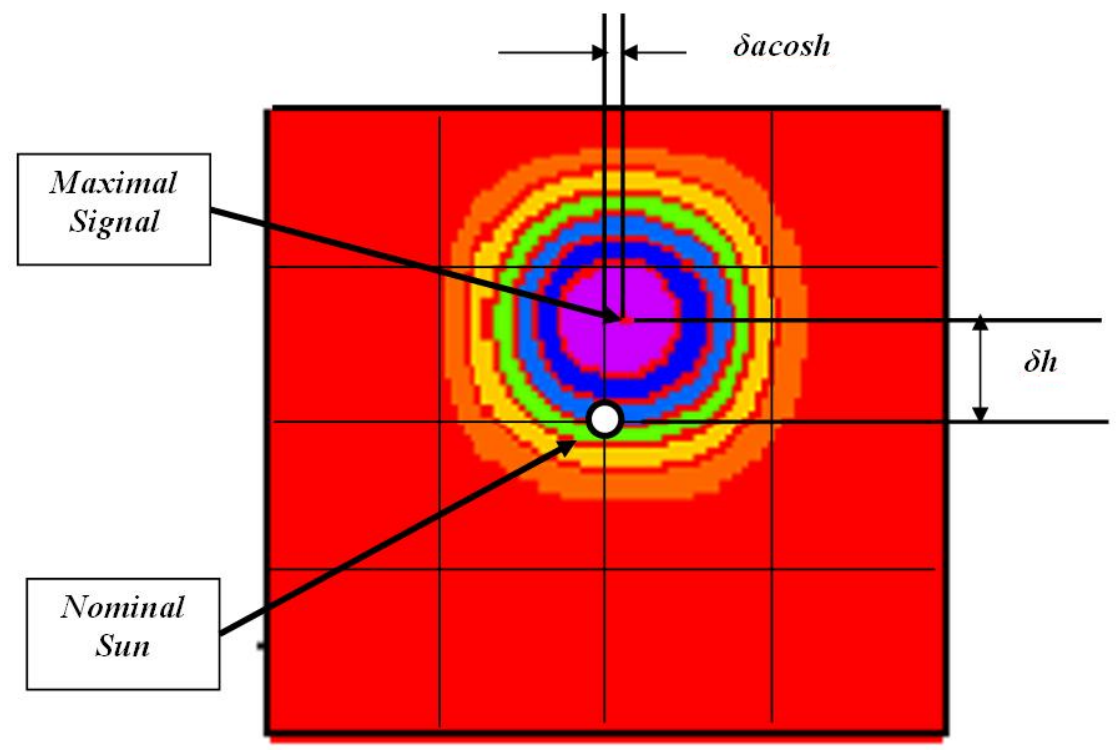

Fig. 4. A typical grid scan: the $5 \times 5$ grid, centered on the nominal Sun, is shown together with the signal density in local coordinates $($ dacosh, $d h)$. The definition of the offsets is illustrated.

The scan of the grid is performed from east to west in lines of increasing elevation, with the SRT pointing nominally to the node of the grid that is being measured. The nominal Sun is at the azimuth and elevation corresponding to the thirteenth grid node. 
The angular distance $d_{i}$ between the true Sun and grid node $i$ at the time when it is being measured is easily calculated by using the relation.

$$
\cos d=\cos \left(h_{1}-h_{2}\right)-\cosh _{1} \cosh _{2}\left[1-\cos \left(a_{1}-a_{2}\right)\right]
$$

which gives the angular separation between the directions $\left(a_{1}, h_{1}\right)$ and $\left(a_{2}, h_{2}\right)$.

Relation 1 reduces to: $d^{2}=\left(h_{1}-h_{2}\right)^{2}+\cosh _{1} \cosh _{2}\left(a_{1}-a_{2}\right)^{2}$

in the limit of small angular separations: locally, one has a Euclidean metric in coordinates (dacosh, dh).

The best Gaussian fit of the measured signals $S_{i}$, integrated over frequency, to a form $A+B \exp \left(-0.5 d_{i}^{2} / \sigma^{2}\right)$ is used to define the SRT offsets in azimuth and elevation, $d a$ and $d h$. In practice, in addition to $d a$ and $d h, A$ and $B$ are free parameters adjusted by the fit while $\sigma$, a measure of the lobe size, is fixed at $2.34^{\circ}$ (see below). The dependence on azimuth of the best fit values associated with each grid scan is illustrated in Fig. 5 left.

\section{III.2. Parameterization}

Two main causes come to mind to explain why $d a$ and $d h$ deviate significantly from zero: a possible tilt of the SRT rotation axis with respect to vertical and zero offsets of the azimuth and elevation scales. A tilt by an angle $\varepsilon_{0}$ in a plane of azimuth $a_{0}$ generates offsets

$$
d a=-\varepsilon_{0} \sin \left(a-a_{0}\right) \tanh \text { and } d h=-\varepsilon_{0} \cos \left(a-a_{0}\right)
$$

Defining $\varepsilon_{1}=\varepsilon_{0} \operatorname{sina}_{0}$ and $\varepsilon_{2}=\varepsilon_{0} \cos a_{0}$, one obtains $d a=\varepsilon_{1} \operatorname{cosatanh}-\varepsilon_{2} \operatorname{sinatanh}$ and $d h=-\varepsilon_{1} \sin a-\varepsilon_{2} \cos a$ where both $\varepsilon_{1}$ and $\varepsilon_{2}$ are small angles.
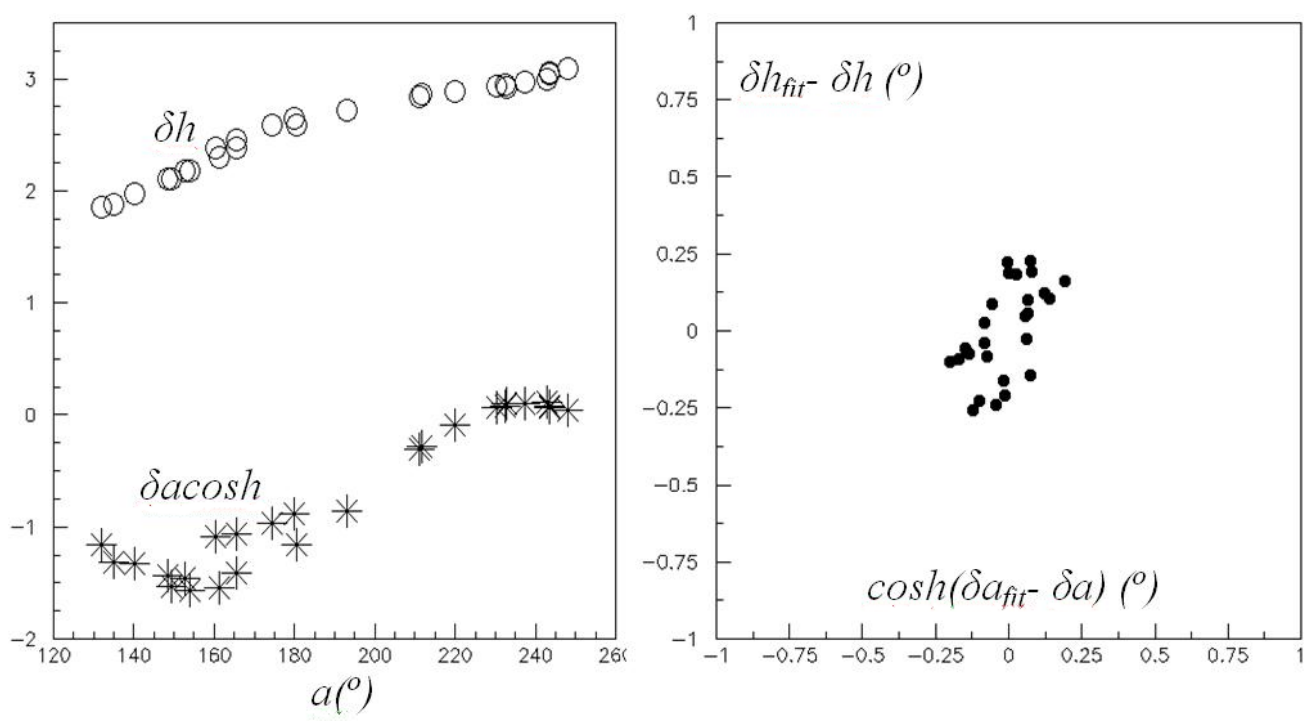

Fig. 5. Left: Dependence on azimuth of the best fit values of dacosh (stars) and $d h$ (circles) associated with each of the 26 grid scans. Right: differences between the measured offsets $(d a \cos h, d h)$ and those calculated by using the best fit values of the $\varepsilon$ parameters (Relation 5). 
We call $\varepsilon_{3}$ the zero offset of the azimuth scale. Rather than defining a zero offset of the elevation scale, it is better to define a zero offset $\varepsilon_{4}$ of the length of the telescopic arm that changes the elevation to which the SRT is pointing. This implies writing the zero offset of the elevation scale as $\varepsilon_{4} \delta h / \delta l$ where the function $\delta h / \delta l$ has been calculated from the geometry of the movement:

$$
\delta h / \delta l=-(0.454-0.0382 f) / g
$$

where $f=51.1-0.391 h-0.00254 h^{2}+0.77 \cdot 10^{-5} h^{3}$ and $g=\sqrt{1-\left(0.956-0.00792 f-0.333 \cdot 10^{-3} f^{2}\right)^{2}}$.

One can now express $d a$ and $d h$ in terms of four small parameters $\varepsilon_{i}$, which define the correction to apply to the nominal pointing direction of the SRT and are related to the azimuth and elevation offsets by the relations:

$$
\begin{gathered}
d a=\varepsilon_{1} \operatorname{cosatan} h-\varepsilon_{2} \operatorname{sinatanh}+\varepsilon_{3} \\
d h=-\varepsilon_{1} \sin a-\varepsilon_{2} \cos a+\varepsilon_{4} \delta h / \delta l
\end{gathered}
$$

Here, the tilt angle is $\varepsilon_{0}=\sqrt{\varepsilon_{1}^{2}+\varepsilon_{2}^{2}}$ and the azimuth of the tilt plane is $a_{0}=$ $\arctan \left(\varepsilon_{1} / \varepsilon_{2}\right)$.

The adequacy of the parameterization is illustrated in Fig. 5 right where the measured offsets $(d a c o s h, d h)$ are compared with those calculated by using the best fit values of the $\varepsilon$ parameters:

$$
\varepsilon_{1}=0.72 \pm 0.05, \varepsilon_{2}=0.95 \pm 0.08, \varepsilon_{3}=-0.15 \pm 0.06, \varepsilon_{4}=-0.98 \pm 0.04
$$

This corresponds to a tilt of $\varepsilon_{0}=1.2^{\circ}$ in the plane of azimuth $37.2^{\circ}$. The rms values of the differences displayed in Fig. 5 right give a measure of the pointing error accuracy: $\cosh \Delta a=0.1^{\circ}, \Delta h=0.15^{\circ}$.

The movement of the SRT dish is controlled by two motors to which one sends pulses [1], each pulse causing a step of $0.42^{\circ}$ in azimuth or $0.85 \mathrm{~mm}$ in the length of the telescopic arm, namely, at an elevation of $45^{\circ}$, an angular step of $\sim 0.3^{\circ}\left(0.30^{\circ}\right.$ in dacosh and $0.13^{\circ}$ in elevation). The above quoted rms values correspond to a third of a motor step in azimuth and one motor step in elevation.

\section{III.3. Drift scans}

Drift scans have been measured in the morning, around noon and in the afternoon of several days between September and December 2011. For each of the 25 drift scans, the telescope was set to point to a given nominal Sun position during the whole duration of the scan, starting approximately half an hour before and ending approximately half an hour after the expected transit of the Sun at that point (Fig. 6). The exact time at which the recorded signal is maximal defines a line in the sky to which the SRT must be pointing. This line is the locus of points for which the distance to the Sun trajectory is minimal at the point where the signal is maximal, namely the major circle normal to the Sun trajectory at the point where the signal is maximal. Calling $h^{*}$ and $a^{*}$ the elevation and azimuth of this point and $h$ and $a$ the elevation and azimuth of the direction to which the SRT is pointing, the projection $d$ on the Sun trajectory of the vector joining these two directions is a measure of the telescope offset. Precisely, calling $(\cos \theta, \sin \theta)$ the unit vector along the Sun trajectory in local coordinates (dacosh,$d h)$,

$$
d=\left(a-a^{*}\right) \cosh \cos \theta+\left(h-h^{*}\right) \sin \theta .
$$




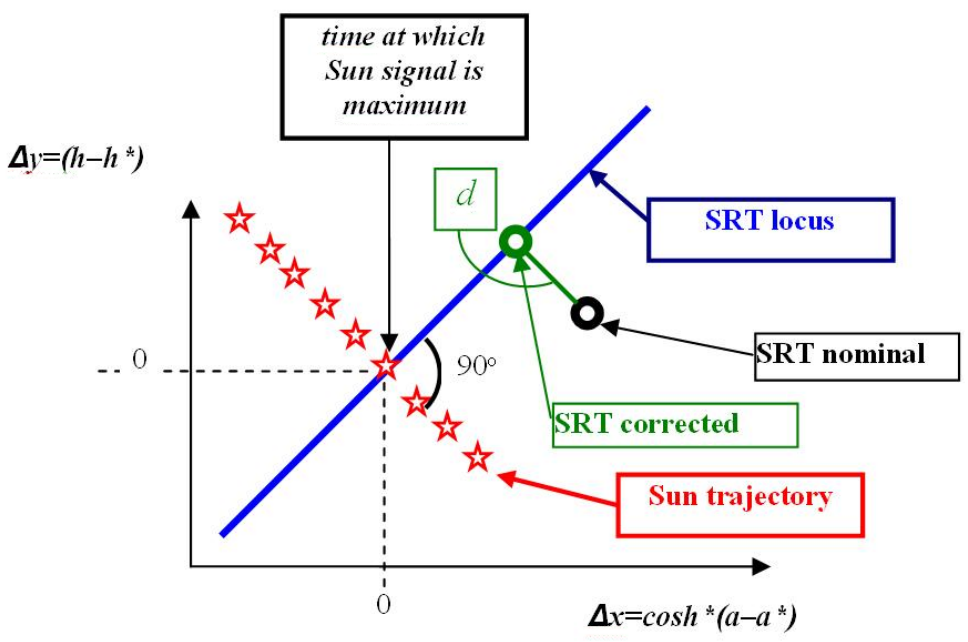

Fig. 6. Schematic of a drift scan across the Sun. The quantity $d$ measured by the scan is indicated.

The rms deviation of $d$ around its mean is $1.2^{\circ}$ before and $0.3^{\circ}$ after applying the pointing corrections given by Relation 5 . This is consistent with the rms values obtained from the grid scans and we shall retain an estimate of $0.3^{\circ}$ for the overall pointing accuracy.

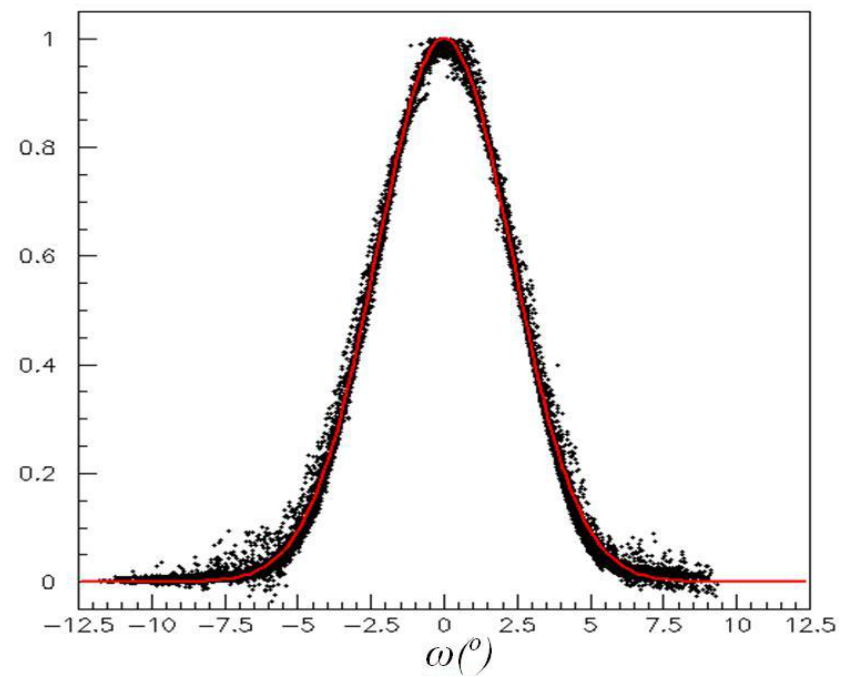

Fig. 7. Dependence of the amplitude of the Sun signal on the angular separation between Sun and SRT. The best Gaussian fit is shown as a line.

Drift scans provide a measurement of the shape of the antenna lobe as illustrated in Fig. 7 where the dependence of the signal, after subtraction of the background and proper normalisation, is displayed as a function of the angular separation $\omega$ between the current Sun position and its position at maximal signal. Indeed, if the signal has a 
Gaussian dependence on the angular separation $\psi$ between Sun and SRT, it also has on $\omega$ as $\psi^{2}-\omega^{2}$ is a constant. The FWHM of the antenna lobe is measured this way to be $5.4 \pm 0.2^{\circ}$, corresponding to $\sigma=2.3 \pm 0.1^{\circ}$.

\section{THE CENTRE OF THE GALAXY: A STRONG 21 CM SIGNAL}

Drift scans across the disk of the Milky Way give evidence for a strong $21 \mathrm{~cm}$ signal. The centre of the Galaxy contains a black hole, Sgr A*, having a mass of some 3 million solar masses; it is a strong radio source [4]. The disk of the Galaxy, and particularly its centre, contain a large number of hydrogen clouds that are the source of the observed 21 cm signal.
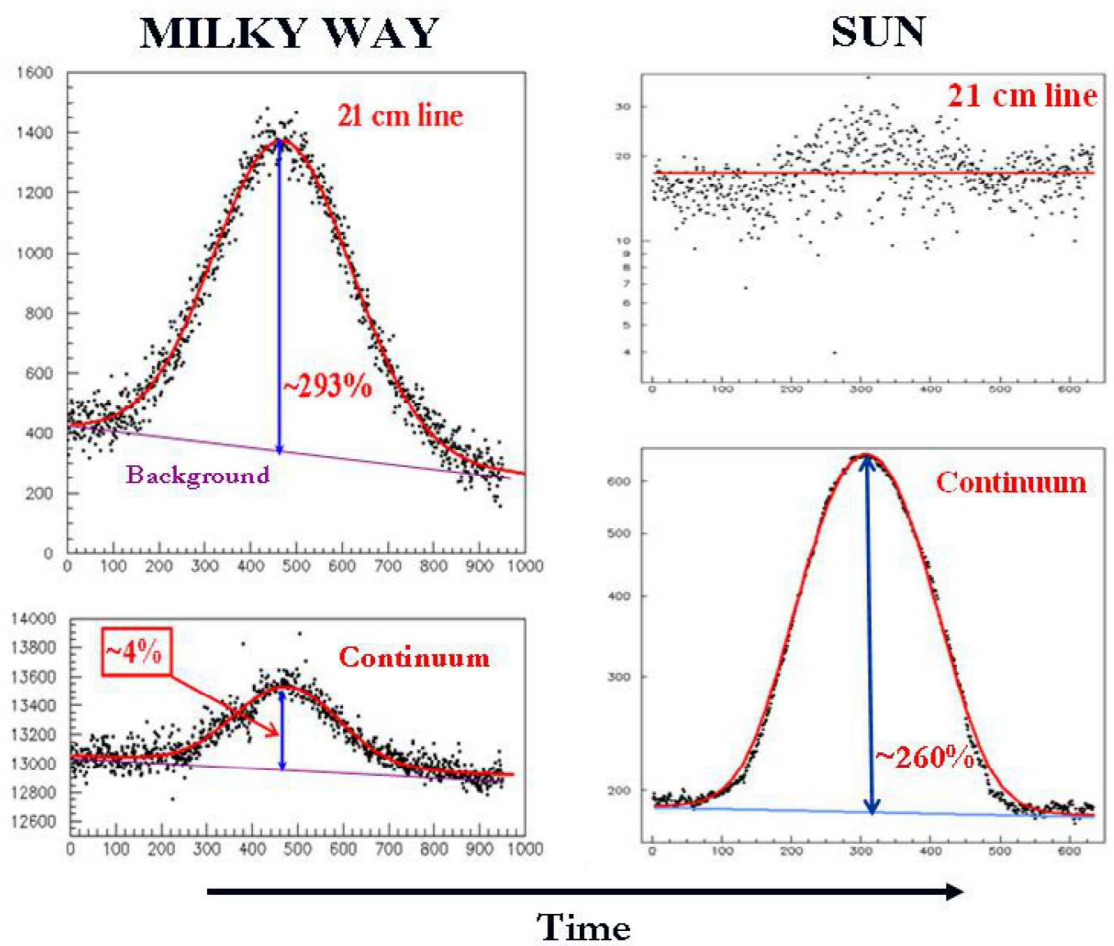

Fig. 8. Drift scans across the centre of the Milky Way (left) and across the Sun (right). The $21 \mathrm{~cm}$ signal (upper panels) and the continuum signal (lower panels) are shown separately. The difference between the Milky Way, dominated by hydrogen clouds, and the Sun, dominated by a hot plasma, is spectacular.

Fig. 8 illustrates such a drift scan and compares it with a drift scan across the Sun. In the former case, there is only a small enhancement of the continuum, due to the much higher density of stars in the direction of Sgr A*, while the $21 \mathrm{~cm}$ signal is nearly tripled due to the presence of hydrogen clouds. In the Sun case, on the contrary, it is the continuum signal that is strongly enhanced, again by a factor of nearly 3 , while the $21 \mathrm{~cm}$ 
signal is essentially unaffected (the small enhancement visible on Fig. 8 is an artefact of our definition of the continuum signal, not going far enough on the tails of the Sun signal). Indeed, there is no enhancement of neutral hydrogen in the direction of the Sun.

\section{A RICH PROGRAMME OF MEASUREMENTS}

The data presented here have provided ample evidence for the excellent performance of the telescope. This opens the way to a series of interesting observations and measurements that will be made in the near future.

The Sun has recently entered a new phase of activity after a long period of quietude [5]. Solar flares are associated with large radio bursts that tend to last longer than the visible flares [6]. Following the Sun radio emission over, say, two revolutions (namely $\sim 2$ months) will make it possible to detect such bursts and to compare them with their counterparts observed at other frequencies of the electromagnetic spectrum. The radio signals observed in both successive revolutions will be compared and correlated with existing solar spots in the field of view.

Drift scans across the disk of the Milky Way, repeated at different galactic longitudes, allow for a precise mapping of the hydrogen clouds in the SRT field of view and measurements of their radial velocities. Indeed, as illustrated in Fig. 9, Doppler shifts of $\sim 0.03 \mathrm{MHz}$ can easily be measured, corresponding to radial velocities of $\sim 7 \mathrm{~km} / \mathrm{s}$, smaller than typical relative radial velocities in the Galaxy.

A careful mapping of neutral hydrogen clouds in the Milky Way disk will not only provide an estimate of the rotation velocity of the Galaxy but also reveal some of its arm structure. The results will be confronted with what can be expected from Kepler laws and an estimate of the amount of dark matter contained in the Milky Way will be given.

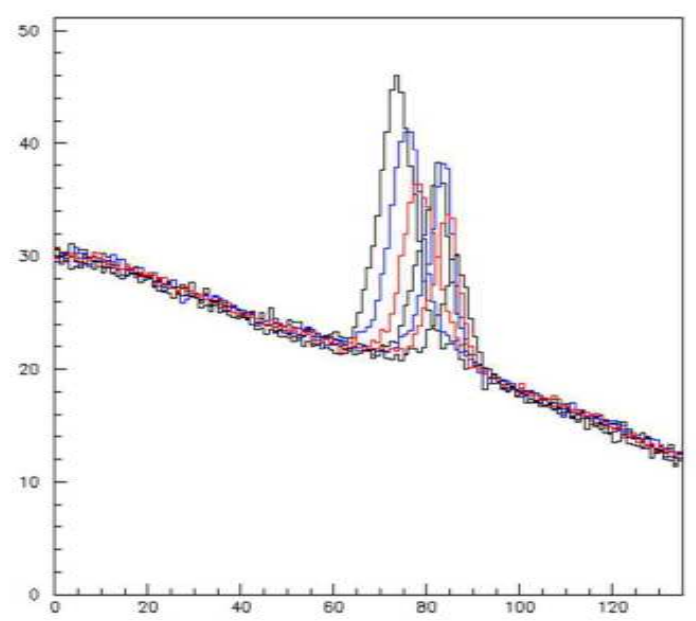

Fig. 9. Frequency spectra showing the sensitivity to different Doppler shifts of the $21 \mathrm{~cm}$ line.

Moreover other radio sources will be observed [7] and their $21 \mathrm{~cm}$ and continuum signals will be measured. These include in particular nearby galaxies such as Andromeda, 
nearby Active Galactic Nuclei such as Cen A [8] and young Super Nova remnants such as Cassiopae and the Crab.

\section{ACKNOWLEDGEMENTS}

We express our deepest gratitude to Dr Alain Maestrini who helped us with the installation and commissioning of the telescope. Valuable measuring instruments have been given to VATLY by the Paris Observatory on his initiative and we thank all those who contributed to it. Financial and/or material support from the Institute for Nuclear Studies and Technology, NAFOSTED, the World Laboratory, Rencontres du Vietnam and Odon Vallet fellowships is gratefully acknowledged.

\section{REFERENCES}

[1] Produced by Custom Astronomical Support Services, Inc.

[2] N.V. Hiep, Presented at the Second Academic Conference on Natural Science for Master and PhD students from Cambodia, Laos, Malaysia and Vietnam, 11-15 October 2011, Vinh, Vietnam.

[3] P. T. Anh, Radio Detection of the Sun, Master thesis presented at the Institute of Physics, Hanoi, September 2010.

[4] Kim Thi Phuong, The Black Hole in the Centre of the Milky Way, Dissertation presented at the Hanoi University of Sciences, Faculty of Physics, 2006, and references therein.

[5] http://www.universetoday.com/10846/predicting-times-for-clear-space-weather.

[6] R. E. Loughhead, J.A. Roberts and M.K. McCabe, The Association of SolarRadio Bursts of Spectral Type III with Chromospheric Flares, Aus. J. Phys. 10 (1957) 483.

[7] R. X. McGee, J.D. Murray and J.A. Milton, Aust. J. Phys. 16 (1963) 136.

[8] Le Thi Huong, Galaxy Collisions as Possible Sources of Ultra High Energy Cosmic Rays, Dissertation presented at the Hanoi National University of Education, 2008, and references therein.

Received 18 March 2012. 